\title{
Peripheral NK Cells Levels Major than 10\% are Associated to Recurrent Pregnancy Loss in Selected Population \\ Di Micco $\mathbf{P}^{*}$ \\ UOC Medicine, Ospedale Fatebenefratelli di Napoli, Italy
}

\begin{abstract}
Recurrent pregnancy loss is a daily intriguing work in women in reproductive age. Differential diagnosis should always be considered because RPL could be related to several clinical condition as chromosomal aberration, endocrine abnormalities, uterine malformations, uterine infections thrombophilia and so on. Immunological abnormalities have also been considered in this clinical setting both as chronic inflammatory disease as abnormal levels of subset of immune cells or antigens. Immunological abnormalities in fact are frequently considered for unexplained RPL although a clear clinical and therapeutic approach actually is not available. Increased levels of peripheral NK cells seem to be a suitable marker to select women with RPL for immune cells abnormalities.
\end{abstract}

\section{Background}

Increased levels of NK cell have been frequently underlined as risk factor for recurrent pregnancy loss (RPL) [1]. This kind of alteration has been found both in women with spontaneous pregnancy and after in vitro fertilization with embryo transfer [2]. On the other hand RPL represents a major health problem with two to three or more losses in up to $5 \%$ of women of reproductive age and it has been considered as one of the most common causes of female infertility [3]

In daily clinical practice it is very difficult to have a real dimension of this kind of alteration because other risk factors for RPL seem to be more frequently diagnosed. Several reports identified several clinical condition and diseases potentially responsible of RPL such as endocrine diseases (e.g., ovarian dysfunction, anovulation, hypopituitarism, diabetes), uterine malformations, genetic alterations (e.g., chromosomal aberrations), hypercoagulable state and thrombophilia (e.g., inherited thrombophilia, antiphospholipid syndrome, combined thrombophilia), chronic inflammatory diseases (in particular systemic lupus erythematosus), infectious diseases and immunological abnormalities [4-6]. However, it has always been difficult to establish a thorough magnitude of immune etiology of RPL because its incidence in each study may be different because of the adopted different inclusion and exclusion criteria. However, also for these other risk factors for RPL it is difficult to establish a clear percentage in the real world population because actually these values are derived from several retrospectives studies in which different adopted inclusion and exclusion criteria were different.

So, because a significant portion of unexplained pregnancy loss is associated with immune etiology other than immunopathological conditions as erythematosus lupus or antiphospolipid syndrome or autoimmune diseases in our outpatients clinic, we performed a retrospective analysis of NK cells peripheral values in women with unexplained RPL in order to have also a cut off levels of NK cells that may suggest a different therapy to escape further miscarriages.

\section{Patients and Methods}

We retrospectively analyzed our records concerning outpatients with recurrent pregnancy loss associated only to increased NKc peripheral levels and compared them to patients with spontaneous pregnancy without extra-troubles to delivery.

Selected patients were 100 for both groups. Patients taking antithrombotic therapies or antibiotics were excluded by this analysis in both groups. NK cells peripheral levels were divided in three subgroups: less than $10 \%, 10-19 \%$ major than $20 \%$. Statistical analysis was based on chi square test, differences were considered to be significant if $\mathrm{p}<0.05$. Statistical analysis was carried out using SPSS statistical software.

\section{Results}

NK cells peripheral levels are increased in patients with RPL. NK cells levels major than $10 \%$ are present in nearly $90 \%$ of patients with RPL while are normal in more than $80 \%$ of women with regular pregnancy and delivery. Interestingly a relevant number of patients showed intermediate levels of NK cells between 10 and 19\% compared to regular pregnancy. Moreover, there is also a six fold increase of patients with RPL with NK cells major than $20 \%$ compared to patients with regular pregnancy. Results are summarized in Table 1.

\section{Discussion}

Although at the beginning of the century several immune dysfunctions have been described in mice with RPL, this topic has been frequently matter of discussion in humans.

In several reports in the Literature in fact an impaired ratio of $\mathrm{T}$ helper lymphocyte type 1 and type 2 or an increased percentage of NK lymphocytes have been associated to RPL [7].

The impairment of immunological functions of a pregnant woman may start from the identification of paternal antigens present on the

\begin{tabular}{|l|c|c|l|}
\hline & $\begin{array}{c}\text { Spontaneous } \\
\text { pregnancy (n100) }\end{array}$ & $\begin{array}{c}\text { Recurrent pregnancy } \\
\text { loss (n100) }\end{array}$ & p \\
\hline NKc peripheral<10\% & 89 & 10 & $<0.05$ \\
\hline NKc peripheral $10-19 \%$ & 9 & 72 & $<0.05$ \\
\hline NKc peripheral>20\% & 2 & 18 & $<0.05$ \\
\hline
\end{tabular}

Table 1: NK cells peripheral levels in women with RPL and regular pregnancy.

*Corresponding author: Di Micco P, UOC Medicine, Ospedale Fatebenefratelli di Napoli, Italy, Tel: 393398 078146; E-mail: pdimicco@libero.it

Received September 26, 2018; Accepted September 29, 2018; Published September 30, 2018

Citation: Micco DP (2018) Peripheral NK Cells Levels Major than 10\% are Associated to Recurrent Pregnancy Loss in Selected Population. J Blood Lymph 8 : 226. doi: 10.4172/2165-7831.1000226

Copyright: (c) 2018 Micco DP. This is an open-access article distributed under the terms of the Creative Commons Attribution License, which permits unrestricted use, distribution, and reproduction in any medium, provided the original author and source are credited. 
fetus and this mechanism is related to the following RPL [8]. Immune functions related to this process are different as migration of immune cells, cytokine production and impaired lymphocytes $\mathrm{T}$ ratio that may increase the number of local and systemic NK cells. Immune cells most frequently associated to migration to uterine district, in fact, are peripheral blood monocytes (PBMc) and Natural Killer cells (NKc). Besides cytokines network, endocrine and paracrine productions of female sexual hormones are also associated to the increase of $\mathrm{NKc}$ in the decidual endometrium during the implantation [9] so giving further alteration to the balance of peripheral NK cells vs. uterine NK cells. This impairment may be recognized as potential responsible of abnormal immune response toward fetus $[9,10]$.

These alterations may be relevant from a clinical point of view too, actually in fact the percentage of unexplained RPL varies from 20 to $35 \%$ and immunological alterations seem to be to most involved. However, as previously underlined, a real magnitude of the increase of peripheral NKc cannot be found actually because available studies show different inclusion and exclusion criteria in described populations. However, in previous studies few indications about the dangerous levels of peripheral NKc have been provided.

\section{In our population}

On the other side besides these several immunological alterations have been identified, little is known from a therapeutic point of view. First of all although the reduction of levels of NKc seems too possible with the use of steroids, none reported an appropriated dose of steroids that can be used in this clinical setting and furthermore none suggested how this therapeutic approach can be monitored during the treatment. So haemocrome with total lymphocytes count and a repetition of peripheral NKc levels have been considered but actually without any type of confirm from a clinical point of view. Thereafter also studies on the prognosis of women with RPL and increased peripheral NKc are treated with steroids or other drugs lacking in the Literature so underling that we are at the beginning of an unsolved problem [11-13].

In conclusion we can assert that immunological abnormalities other than immunological systemic disease, should be involved and investigated in the pathophysiology of RPL in particular for cases with unexplained RPL, however appropriated clinical strategies and studies to identify, to treat and to monitor this clinical problem should be better planned in next years on larger populations.

\section{References}

1. Di Micco P, Strina I, Lodigiani C (2018) NK cells and unexplained recurrent pregnancy loss, a well-known but rarely considered association. J Blood Disord Symptoms Treat 2: e05-06

2. Thum MY, Bhaskaran S, Abdalla HI, Ford B, Sumar N, et al. (2004) An increase in the absolute count of $\mathrm{CD} 56^{\mathrm{dim}} \mathrm{CD} 16^{+} \mathrm{CD} 69^{+} \mathrm{NK}$ cells in the peripheral blood is associated with a poorer IVF treatment and pregnancy outcome. Human Reprod 19: 2395-2400.

3. Sarig G, Younis JS, Hoffman R, Lamir N, Blumenfeld Z, et al. (2002) Thrombophilia is common in women with idiopathic pregnancy loss and is associated with late pregnancy wastage. Fertil Steril 77: 342-347.

4. Eldor A (2001) Thrombophilia, thrombosis and pregnancy. Thromb Haemost 86: 104-101.

5. Carp H, Salomon O, Seidman D, Dardik R, Rosenberg N, et al. (2002) Prevalence of genetic markers for thrombophilia in recurrent pregnancy loss. Hum Reprod 17: 1633-1637.

6. Di Micco P, D'Uva M, Strina I, De Placido G (2010) Recurrent pregnancy loss and thrombophilia. J Clin Med Res 2: 18-22.

7. Kwak-Kim J, Park JC, Ahn HK, Kim JW, Gilman-Sachs A (2010) Immunological modes of pregnancy loss. Am J Reprod Immunol 63: 611-623.

8. Laird SM, Tuckerman EM, Cork BA, Linjawi S, Blakemore Al, et al. (2003) A review of immune cells and molecules in women with recurrent miscarriages. Hum Reprod Update 9: 163-174.

9. Dosiou C, Giudice KC (2005) Natural killer cells in pregnancy and recurrent pregnancy loss: endocrine and immunologic perspectives. Endocrin Rev 26: 44-62.

10. Han G, Chen Y, Xu J, Xie X, Yu D, et al. (2017) The regulation of ovary and conceptus on the uterine natural killer cells during early pregnancy. Reprod Biol Endocrinol 15: 73.

11. Zhu L, Aly M, Wang H, Karakizlis H, Weimer R, et al. (2018) Increased natura killer cell subestes with inhibitory cytokines and inhibitory surface receptors in patients with recurrent miscarriage and decreased or normal subsets in kidney transplants recipients late post transplant. Clin Exp Immunol 193: 241-254.

12. Chen X, Mariee N, Jiang L, Liu Y, Wang CC, et al. (2017) Measurement of uterine natural killer cell percentage in periimplantation endometrium from fertile women and women with recurrent reproductive failure: establishment of a reference range. Am J Obstetric Gynecol 217: 680-686.

13. Sokal RR, Rohlf FJ (1992) Biometry: the principles and practice of statistics in biological research (3rd edn). W. H. Freeman, New York, USA. 